\title{
Inpatient Mortality and 30-Day Readmission Rates Associated with Troponin Testing in Patients without Acute Myocardial Infarction
}

R. Jay Widmer, MD, PhD; Gibbs Wilson, MD; Travis Haneke, MD; Mary Lee, DO; Jerry Fan, MD; Arthur Davis, MD; Ethan Fry, DO; Kevin Kurian, MD; Jonathan Urbanczyk, DO; Jordan Potter, DO; Matthew McMahon, MD; Bryce Rhodehouse, DO; Joon J. Song, PhD; and Jeffrey B. Michel, MD

Objective: Troponin values above the threshold established to diagnose acute myocardial infarction (AMI; >99th percentile) are commonly detected in patients with diagnoses other than AMI.The objective of this study was to compare inpatient mortality and 30-day readmission rate in patients with troponin I (Tnl) above and below the 99th percentile in those with type I AMI and type 2 myocardial injury.

Methods: Between January I, 2016 and December 3I, 2016, there were 56,895 inpatient hospitalizations; of these $14,326(25.2 \%)$ patients received troponin testing. We evaluated mortality and readmissions in the entire cohort based on the primary discharge International Classification of Diseases, Tenth Edition (ICD-I0) diagnosis and grouped into type I AMI versus other diagnoses comprising the type $2 \mathrm{AMI}$ group (including ICD-10 codes for congestive heart failure, sepsis, and other). Among those with $\mathrm{Tnl}$ drawn, we evaluated in-hospital mortality and 30-day readmissions based on troponin values $>99$ th percentile $(\geq 0.1 \mathrm{ng} / \mathrm{ml})$.

Results: Among the entire cohort, the inpatient mortality rate was significantly higher in those with $\mathrm{Tnl}$ testing $(5.0 \%, 95 \% \mathrm{Cl} 4.6 \%-5.3 \%)$ compared to those without testing $(0.7 \%, 95 \% \mathrm{Cl} 0.6 \%-0.7 \%$, $P<0.01)$. In the tested cohort 3,743 (26\%) patients had troponin levels above the 99th percentile $(>0.1 \mathrm{ng} / \mathrm{ml})$, and $10,583(74 \%)$ had troponin levels below the 99th percentile $(\leq 0.1 \mathrm{ng} / \mathrm{ml})$. Comparing type 2 AMI with type I AMI and troponin testing, Tnl values $\geq 0.1 \mathrm{ng} / \mathrm{ml}$ were associated with higher inpatient mortality (11.6\% vs. $3.9 \%$ ) and 30 -day readmission rates (16.9\% vs. $10.7 \%$ ).

Conclusions: A higher inpatient mortality and 30-day readmission rates were found in patients with type 2 AMI compared to type I AMI group.

Keywords: 30-day Readmission; Inpatient Mortality; Troponin; Type 2 MI

Corresponding Author: R. Jay Widmer, MD, PhD, Division of Cardiology, Scott \& White Heart Memorial Hospital, Baylor Scott \& White Health, 240I 3 Ist Street,

Temple,TX 75608, Email: Robert.Widmer@BSWHealth.org
Received: June 22, 2019

Ist Revision: August 26, 2019

2nd Revision: October 4, 2019

3rd Revision: October 26, 2019

Accepted: January 2, 2020

doi: $10.3121 / \mathrm{cmr} .2020 .1513$ 
$\mathrm{T}$ The current Universal Definition of acute myocardial infarction (AMI) establishes the explicit diagnosis when patients with symptoms of possible AMI have a rise or

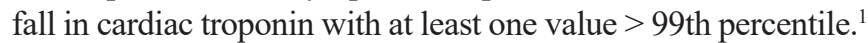
Troponin testing has become widespread and now includes large numbers of patients with AMI secondary to plaque rupture (type $1 \mathrm{AMI}$ ) and those with troponin elevation from clinical conditions whereby myocardial oxygen supply is insufficient to meet elevated demands (type $2 \mathrm{AMI}$ ), such as demand ischemia, heart failure, spontaneous coronary artery dissection/vasospasm, hypertensive emergency, or other similar conditions. The negative predictive value of troponin testing is high, allowing physicians to use the test primarily to exclude, rather than diagnose, AMI. However, the testing of large numbers of patients without AMI has contributed to poor positive predictive value (PPV) of those with type $1 \mathrm{AMI}^{2}$ Data from our system have demonstrated a PPV $<30 \%$ in actual clinical practice with the majority of troponin values $>$ 99th percentile not associated with type 1 AMI.,3

Troponin elevations are known to occur in conditions not associated with coronary plaque rupture including sepsis, pneumonia, stroke, atrial fibrillation, and congestive heart failure $(\mathrm{CHF})^{4}$ due to increased, yet unmet, myocardial oxygen demand resulting in a type 2 AMI. Discovery of elevated troponin levels may influence clinical decision-making regarding the use of "downstream" cardiac imaging including echocardiography and coronary angiography. Previous research in a large tertiary care center reports a low rate of patients with AMI along with some racial disparities in troponin testing and outcomes. ${ }^{5}$ Furthermore, patients with sepsis demonstrated increased utilization of electrocardiography, echocardiography, and cardiac catheterization in patients with troponin levels $>$ 99th percentile. $^{2}$ There is also interest in troponin as a prognostic marker for patient outcomes, particularly with regard to type 2 AMI that stem from CHF exacerbations ${ }^{6}$ or patients admitted with sepsis. ${ }^{7}$ We hypothesized that troponin values $\geq 0.1 \mathrm{ng} / \mathrm{ml}$ seen in type 2 AMI would be associated with the highest rates of inpatient mortality and 30-day readmission among tested, hospitalized patients.

\section{Methods}

All inpatient admissions within a large healthcare system comprising nine hospitals in Texas were evaluated for calendar year 2016 (January 1, 2016-December 31, 2016). The study was granted Institutional Review Board approval and did not require approval by the Ethics Board. Criteria for patient inclusion were adult ( $>18$ years-of-age) inpatient hospitalizations with an admission date from January 1, 2016 to December 31, 2016. When multiple admissions occurred for an individual patient, only the first admission within the study period was included in the analysis of outcomes. Demographic variables evaluated for

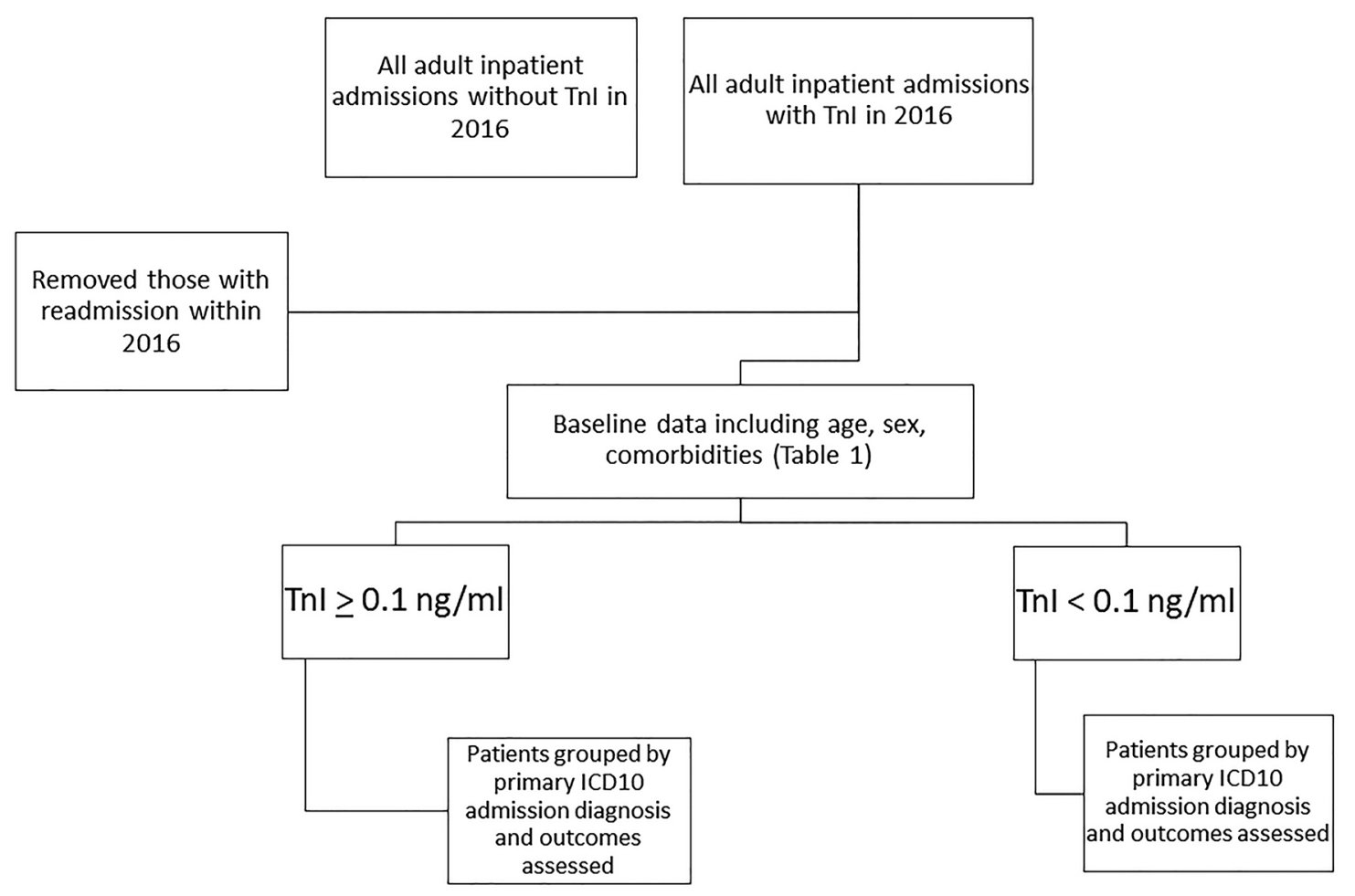

Figure 1. Study designed to divide inpatients into those who did and who did not undergo troponin I (Tnl) testing. Those with Tnl testing were further grouped according to $\mathrm{Tnl}$ below or above the 99th percentile threshold $(\geq 0.1 \mathrm{ng} / \mathrm{ml})$ and then by primary etiology for the hospital admission based on ICD 10 code. Mortality and 30-day readmission data were captured from the electronic health record and data analyzed. 
all admissions during the study period included age, sex, creatinine, and the presence of hypertension, hyperlipidemia, diabetes, smoking, and prior kidney disease. Patients were first grouped into those who underwent at least one troponin test and those not tested (Figure 1).

Admissions were then grouped based on primary International Classification of Diseases, Tenth Edition (ICD-10) diagnosis. Encounters with discharge diagnoses, "ICD-10 I21" were designated as type 1 AMI. The type 2 AMI cohort was created by subtracting the type 1 AMI group from the remainder of the patients. Among the type $2 \mathrm{MI}$ cohort, further delineation was made based on ICD codes for CHF (ICD-10 I50), sepsis (ICD10 A41), and "Other" being those without the diagnoses of AMI, $\mathrm{CHF}$, or sepsis.

The troponin I (TnI) assay (AccuTnI+3, Beckman Coulter vs. Elecsys Troponin I Assay, Roche) is used exclusively within this hospital system with results $\geq 0.1 \mathrm{ng} / \mathrm{ml}$ representing the 99th percentile threshold for elevation. In those patients who underwent troponin testing, we further delineated those with a $\mathrm{TnI}$ value $\geq 0.1 \mathrm{ng} / \mathrm{ml}$ and those with a highest reported $\mathrm{TnI}$ value $<0.1 \mathrm{ng} / \mathrm{ml}$ (Figure 1). The primary endpoint of the study was inpatient mortality, and the secondary endpoint was 30-day readmission with both endpoints abstracted and adjudicated through a review of the electronic health record.

\section{Statistical Analysis}

Baseline demographics for the entire cohort are presented as numeric counts, averages, percentages, and 95\% confidence intervals (Table 1). The two-proportion z-test was used to compare the difference between groups and the corresponding $P$ value was computed. All tests indicate statistical significance at the $5 \%$ significance level.

\section{A. Troponin Testing and Mortality}

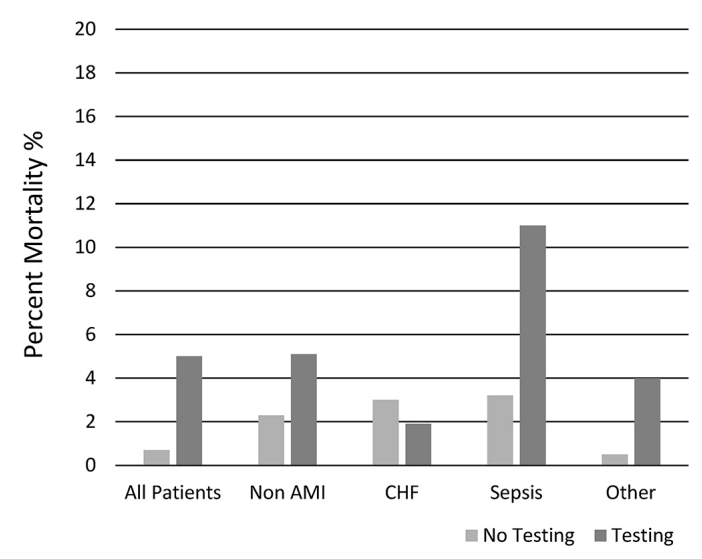

\section{Results}

A total of 14,326 unique admissions were associated with troponin testing when subsequent hospitalizations were excluded from the analysis. Baseline demographics associated with TnI testing are displayed in Table 1.

The overall inpatient mortality rate for all admissions was $1.7 \%$ $(994 / 56,895)$. Mortality for all patients with troponin testing was $5.0 \%(713 / 14,326)$, while mortality for patients without testing was $0.7 \%(281 / 42,569)$ (Table 2$)$. When troponin was assayed and values $\geq 0.1 \mathrm{ng} / \mathrm{ml}$ were detected, mortality was $9.2 \%$ $(346 / 3,743)$. When troponin values $<0.1 \mathrm{ng} / \mathrm{ml}$ were detected, mortality was $3.5 \%(367 / 10,583)$ (Table 3$)$.

For patients with a primary diagnosis of type 1 AMI, overall mortality was $3.9 \%(45 / 1,151)$. Figure $2 \mathrm{~A}$ demonstrates percent mortality in patients tested for TnI and those who did not undergo testing. Mortality was significantly higher in patients who underwent troponin testing compared to those who did not undergo TnI testing (4.98\% vs $0.67 \%$, respectively, $P<0.01)$. Additionally, in patients with any troponin testing, type 2 AMI patients were associated with a higher mortality rate compared to those with type 1 AMI (5.1\% vs $4.0 \%)$. Higher mortality was noted in patients who underwent TnI testing in the sepsis and "other" subsets of type 2 AMI, but not within the CHF cohort (Table 2).

Among those admitted for type 2 AMI, there was a significantly higher percent mortality in those with $\mathrm{TnI} \geq 0.1 \mathrm{ng} / \mathrm{ml}$ compared with type 2 AMI and non-elevated troponin (11.6\% vs 3.5\%, respectively, $P<0.01)$. In CHF patients, $\mathrm{TnI} \geq 0.1 \mathrm{ng} / \mathrm{ml}$ was associated with a mortality rate of $2.7 \%$ (13/476), while mortality rate with non-elevated troponin was $1.5 \%(21 / 1,362$, $P=0.1)$. In "other" patients, $\mathrm{TnI} \geq 0.1 \mathrm{ng} / \mathrm{ml}$ was associated

\section{B. Troponin Values and Mortality}

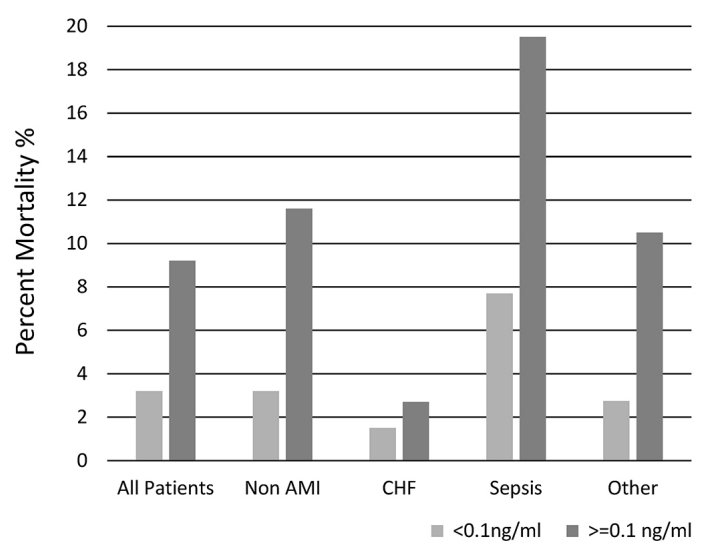

Figure 2. Association between troponin (Tnl) testing, values and inpatient mortality. Mortality rates were determined for all inpatients, as well as for inpatients without a primary diagnosis of AMI (type 2 AMI) including those with primary diagnoses of CHF, Sepsis, and Other. Comparison was then made between patients tested and not tested for Tnl (A). Inpatient mortality was evaluated for inpatients with a Tnl value $\geq 0.1 \mathrm{ng} / \mathrm{ml}$ (99th percentile for assay) (B). 
Table 1. Baseline demographics for inpatients with and without troponin (Tnl) testing

\begin{tabular}{llll}
\hline & $\begin{array}{l}\text { AMI } \\
\text { Troponin } \geq 0.1\end{array}$ & $\begin{array}{l}\text { No AMI } \\
\text { Troponin } \geq 0.1\end{array}$ & $\begin{array}{l}\text { No AMI } \\
\text { Troponin }<0.1\end{array}$ \\
\hline Age (Years) & $65.82(65.01,66.63)$ & $69.94(69.38,70.50)$ & $68.57(68.30,68.84)$ \\
Male & $0.61(0.58,0.54)$ & $0.51(0.49,0.53)$ & $0.47(0.46,0.48)$ \\
Female & $0.39(0.36,0.42)$ & $0.49(0.47,0.51)$ & $0.53(0.52,0.54)$ \\
HTN & $0.36(0.33,0.39)$ & $0.54(0.52,0.56)$ & $0.42(0.41,0.43)$ \\
Hyperlipidemia & $0.86(0.84,0.88)$ & $0.69(0.67,0.70)$ & $0.67(0.66,0.68)$ \\
Type 1 DM & $0.03(0.02,0.03)$ & $0.03(0.02,0.03)$ & $0.03(0.03,0.03)$ \\
Type 2 DM & $0.46(0.43,0.49)$ & $0.45(0.43,0.47)$ & $0.43(0.42,0.43)$ \\
Smoking & $0.64(0.62,0.67)$ & $0.60(0.58,0.62)$ & $0.59(0.58,0.60)$ \\
Chronic Kidney & $0.49(0.47,0.51)$ & $0.37(0.37,0.38)$ \\
Creatinine & $0.27(0.25,0.30)$ & $2.04(1.97,2.11)$ & $1.46(1.43,1.48)$ \\
Total number & $1.44(1.35,1.53)$ & 3323 & 14045 \\
\hline
\end{tabular}

Statistics are provided as "mean (95\% confidence interval)" for age and creatinine, and "percentage (95\% confidence interval)" for all other data.

Abbreviations: $\mathrm{AMI}=$ acute myocardial infarction; HTN = hypertension; DM = diabetes mellitus.

with a mortality rate of $10.5 \%(146 / 1,386)$, while mortality rate with non-elevated troponin was $2.8 \%(202 / 7,339, P<0.01)$ (Figure 2B).

The overall 30-day readmission rate for all admissions was 9.1\% (5,203/56,895). Figure 3A and Table 4 demonstrate 30-day readmission data in patients who underwent TnI testing. Figure 3B and Table 5 demonstrate 30 -day readmission data in patients based on their TnI value. In type 1 AMI patients with any troponin testing, 30-day readmission was $11.0 \%(130 / 1,187)$. Among all patient groups, patients who underwent TnI testing had a significantly higher rate of 30-day readmissions compared to patients without TnI testing (15.0\% vs $7.2 \%$ respectively, $P<0.01)$. Specifically, among type 2 AMI patients who underwent TnI testing, there were significantly higher rates of 30-day readmissions compared to type 2 AMI patients not undergoing TnI testing (15.4\% vs
$7.2 \%, P<0.01)$. There was no significant difference in 30 -day readmission rates in those patients admitted for $\mathrm{CHF}$ exacerbation regardless of troponin testing (18.2\% vs $18.0 \%$, $P=0.8)$.

Regarding TnI level, the rate of 30-day readmissions among those with $\mathrm{TnI} \geq 0.1 \mathrm{ng} / \mathrm{ml}$ was comparable to those with $\mathrm{TnI}$ $<0.1 \mathrm{ng} / \mathrm{ml}$ in all patient subsets (15.0 vs $16.2 \%, P=0.09)$. Similarly, the rates of 30-day readmission in type 2 AMI were comparable in patients with $\mathrm{TnI} \geq 0.1 \mathrm{ng} / \mathrm{ml}$ and in patients with type 2 AMI and normal TnI values (16.9\% vs $16.2 \%$, $P=0.38$ ). Additionally, no significant differences were observed in the 30-day readmission rate in patients admitted with CHF (21.4\% vs $18.0 \%$, respectively, $P=0.1)$ or sepsis (12.7\% vs $14.2 \%$, respectively, $P=0.34$ ) regardless of $\mathrm{TnI}$ elevation versus non-elevation (Figure $3 \mathrm{~B}$ and Table 4 ).

Table 2. Rates of inpatient mortality among inpatients

\begin{tabular}{|c|c|c|c|c|c|c|c|c|c|}
\hline & Tested & Mortality & $\begin{array}{l}\text { Mortality } \\
\text { Rate }\end{array}$ & $95 \% \mathrm{Cl}$ & $\begin{array}{c}\text { Not } \\
\text { Tested }\end{array}$ & Mortality & $\begin{array}{l}\text { Mortality } \\
\text { Rate }\end{array}$ & $95 \% \mathrm{Cl}$ & $P$ value \\
\hline All Patients & 14,326 & 713 & $4.98 \%$ & $0.36 \%$ & 42,569 & 281 & $0.66 \%$ & $0.08 \%$ & $<0.01$ \\
\hline Type 1 AMI & 1187 & 47 & $3.96 \%$ & $1.11 \%$ & 48 & 3 & $6.25 \%$ & $6.85 \%$ & NS \\
\hline Type 2 AMI & 13,139 & 666 & $5.07 \%$ & $0.38 \%$ & 42,521 & 278 & $0.65 \%$ & 0.08 & $<0.01$ \\
\hline CHF & 1838 & 34 & $1.85 \%$ & $0.62 \%$ & 266 & 8 & $3.01 \%$ & $2.05 \%$ & $(0.2)$ \\
\hline Sepsis & 2576 & 284 & $11.03 \%$ & $1.21 \%$ & 2021 & 64 & $3.17 \%$ & $0.76 \%$ & $<0.01$ \\
\hline Other & 8725 & 348 & $3.99 \%$ & $0.41 \%$ & 40,234 & 206 & $0.51 \%$ & $0.16 \%$ & $<0.01$ \\
\hline
\end{tabular}

Groups included all patients, those with the diagnosis of type 1 or type 2 acute myocardial infarction (AMI). Those with type 2 $\mathrm{AMI}$ were further broken down by the primary diagnoses of congestive heart failure (CHF), Sepsis, and Other (Non-AMI/CHF/ Sepsis). Note, very small numbers of AMI patients had no testing and these patients had ST-Elevation Myocardial Infarction had (STEMI), making statistical analysis and comparison between groups unreliable. $(95 \% \mathrm{Cl}=95 \%$ confidence interval, $\mathrm{NS}=$ Not statistically significant). 
Table 3. Mortality rates for inpatients tested for troponin

\begin{tabular}{llllllllll}
\hline & $\begin{array}{l}\text { Troponin } \\
\geq \mathbf{0 . 1}\end{array}$ & Mortality & $\begin{array}{l}\text { Mortality } \\
\text { Rate }\end{array}$ & $\mathbf{9 5 \%} \mathbf{C l}$ & $\begin{array}{l}\text { Troponin } \\
<\mathbf{0 . 1}\end{array}$ & Mortality & $\begin{array}{l}\text { Mortality } \\
\text { Rate }\end{array}$ & $\mathbf{9 5 \%} \mathbf{~ C l}$ & $\boldsymbol{P}$ value \\
\hline All Patients & 3,743 & 346 & $9.24 \%$ & $0.93 \%$ & 10,583 & 367 & $3.47 \%$ & $0.35 \%$ & $<0.01$ \\
Type 1 AMI & 1,151 & 45 & $3.9 \%$ & $1.12 \%$ & 36 & 2 & $5.56 \%$ & $31.3 \%$ & $\mathrm{NS}$ \\
Type 2 AMI & 2,592 & 301 & $11.61 \%$ & $1.23 \%$ & 10,547 & 365 & $3.46 \%$ & $0.35 \%$ & $<0.01$ \\
CHF & 476 & 13 & $2.73 \%$ & $1.46 \%$ & 1,362 & 21 & $1.54 \%$ & $0.65 \%$ & 0.1 \\
Sepsis & 730 & 142 & $19.45 \%$ & $2.87 \%$ & 1,846 & 142 & $7.69 \%$ & $1.22 \%$ & $<0.01$ \\
Other & 1,386 & 146 & $10.53 \%$ & $1.62 \%$ & 7,339 & 202 & $2.75 \%$ & $0.37 \%$ & $<0.01$ \\
\hline
\end{tabular}

Groups included all patients, those with and without a primary diagnosis of type 1 acute myocardial infarction (AMI), and those with primary diagnoses of type 2 AMI with the subsets of congestive heart failure (CHF), Sepsis and Other (Non-AMI/ CHF/Sepsis). (95\% Cl = 95\% confidence interval, NS = Not statistically significant).

\section{Discussion}

These findings build on previous findings demonstrating high use of healthcare resources among those patients who undergo TnI testing and the potential negative impact on overall outcomes-specifically mortality and 30-day readmission rates, when examining first admissions of individual patients within the defined study period. In this hypothesis-generating observational data, those who had TnI testing had substantially higher rates of 30-day readmissions among all patients, specifically those with type 2 AMI. Mortality in patients with type 1 AMI was lower than in patients with elevated TnI secondary to type 2 AMI caused by sepsis or "other" etiology. Interestingly, elevated TnI levels were associated with increased inpatient mortality and 30-day readmission rates in all groups except $\mathrm{CHF}$. These findings suggest type 2 AMI portends a worse overall prognosis.

Overall, these data appear consistent with recent reports from other larger observational studies from similar cohorts with regard to risk factors such as age, elevated creatinine, any smoking history, hypertension, diabetes, and/or hyperlipidemia. ${ }^{8,9}$ For this analysis, we included all inpatient admissions with troponin testing, since patient demographics are known to influence readmission. Not surprisingly, we found patients with more comorbidities carry a higher rate of TnI testing, higher rate of positive TnI results, increased inpatient mortality, and higher 30-day readmission rates.
A. Troponin Testing and 30 Day Readmission

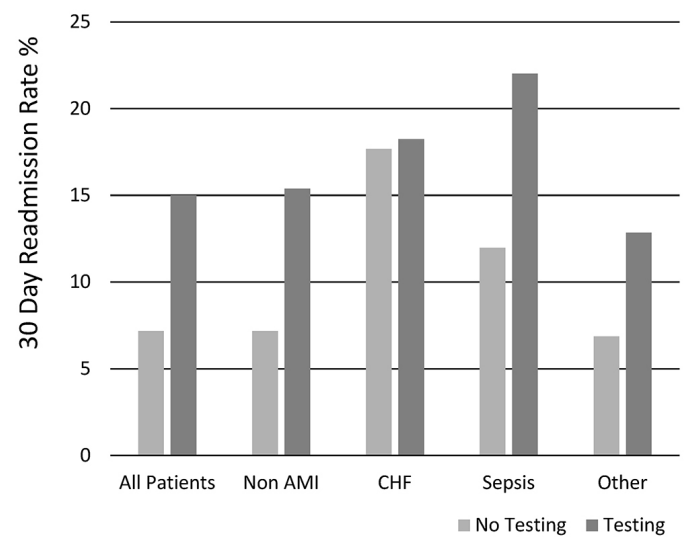

B. Troponin Values and 30 Day Readmission

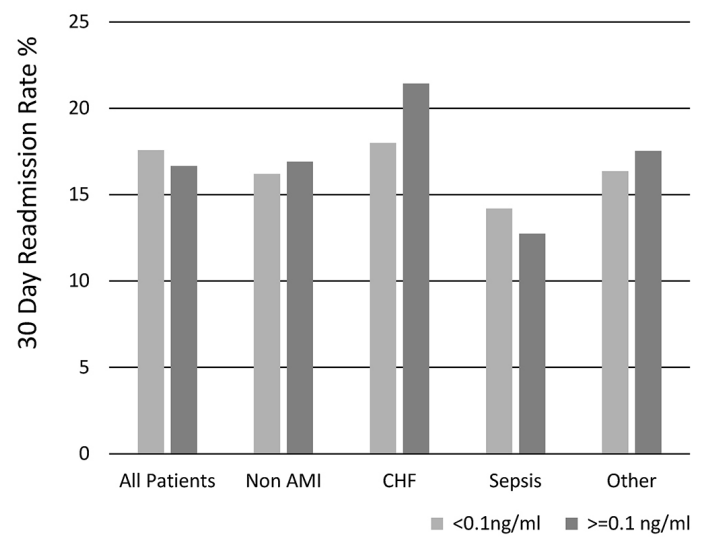

Figure 3. The association between troponin testing, values and 30-day readmission. 30-day readmission rates were determined for all inpatients, as well as for inpatients without a primary diagnosis of AMI (Non-AMI), those with primary diagnoses of CHF and Sepsis, and inpatients without a primary diagnosis of AMI, CHF or Sepsis (Other). Comparison was then made between patients tested and not tested for troponin (A). Troponin testing was associated with statistically significant $(P<0.01)$ greater 30-day readmission rates for all groups except CHF. 30-day readmission rates were evaluated for inpatients with a troponin value $\geq 0.1 \mathrm{ng} /$ $\mathrm{ml}$ (99th percentile for assay) (B). A troponin value $\geq 0.1 \mathrm{ng} / \mathrm{ml}$ was associated with a statistically significant $(P<0.01)$ higher 30 -day readmission rates in all groups except sepsis. For comparison, the 30-day readmission rate was $15.5 \%$ for AMI with no significant difference seen between testing or troponin $\geq 0.1 \mathrm{ng} / \mathrm{ml}$. 
Table 4. 30-day readmission based on troponin testing

\begin{tabular}{|c|c|c|c|c|c|c|c|c|c|}
\hline & $\begin{array}{l}\text { Tested } \\
\text { (n) }\end{array}$ & $\begin{array}{l}\text { 30-day } \\
\text { Readmit } \\
\text { (n) }\end{array}$ & $\begin{array}{l}\text { Readmit } \\
\text { Rate }\end{array}$ & $95 \% \mathrm{Cl}$ & Not Tested & $\begin{array}{l}\text { 30-day } \\
\text { Readmit }\end{array}$ & $\begin{array}{l}\text { Readmit } \\
\text { Rate }\end{array}$ & $95 \% \mathrm{Cl}$ & $\begin{array}{l}P \\
\text { value }\end{array}$ \\
\hline All Patients & 14,326 & 2151 & $15.01 \%$ & $1.39 \%$ & 42,569 & 3052 & $7.17 \%$ & $0.88 \%$ & $<0.01$ \\
\hline Type 1 AMI & 1187 & 130 & $10.96 \%$ & $5.07 \%$ & 48 & 3 & $6.25 \%$ & $26.8 \%$ & NS \\
\hline Type 2 AMI & 13,139 & 2021 & $15.38 \%$ & $1.45 \%$ & 42,521 & 3048 & $7.17 \%$ & $1.23 \%$ & $<0.01$ \\
\hline CHF & 1838 & 335 & $18.23 \%$ & $3.74 \%$ & 266 & 47 & $17.67 \%$ & $9.91 \%$ & $\begin{array}{l}\text { NS } \\
(0.8)\end{array}$ \\
\hline Sepsis & 2576 & 567 & $22.01 \%$ & $3.01 \%$ & 2021 & 242 & $11.97 \%$ & $3.84 \%$ & $<0.01$ \\
\hline Other & 8725 & 1119 & $12.83 \%$ & $1.83 \%$ & 40,234 & 2759 & $6.86 \%$ & $0.91 \%$ & $<0.01$ \\
\hline
\end{tabular}

Groups included all patients, those with and without a primary diagnosis of type 1 acute myocardial infarction (AMI), and those with primary diagnoses of type 2 AMI and the type 2 AMI subsets of CHF, Sepsis and Other (Non-AMI/CHF/Sepsis). (Readmit = readmission; $\mathrm{NS}=$ Not statistically significant $)$.

There is a noted strong link between troponin values and overall prognosis. ${ }^{8}$ We see similar findings here, noting that in nearly all categories of type 2 AMI except for CHF, TnI elevation is a poor prognostic marker in terms of mortality and 30-day readmission. Interestingly, even patients with TnI testing had worse outcomes on the whole. The finding that testing itself correlates with mortality suggests patient selection plays an important role in previously reported associations between elevated troponin values and patient outcomes. The more frequently a population is tested, the more likely it becomes to detect elevated values, and testing itself cannot be expected to cause mortality. A physician preference to test sicker patients likely underlies this observation. While the relative contribution of patient selection for testing and the finding of an elevated troponin is difficult to quantify, our data indicate both are important and highlight that prognosis attributed to elevated troponin values results from both physician acumen and assay results.
Our data set analyzes outcomes based on TnI; however, the implications of these findings on more heterogeneous and comorbid populations is less well known. Perhaps, higher thresholds should be put into place for various patient populations such as those with diabetes, chronic kidney disease (CKD), and other diagnoses prone to microvascular disease to improve the specificity of diagnosing type 1 AMI. Furthermore, data of these outcomes on patients in whom TnI testing was undertaken underscore the importance of interpreting TnI testing judiciously, as our previous work demonstrates a high utilization of downstream testing. ${ }^{2}$ We note a three-fold increase in echocardiography in whom troponin is obtained and noted to be abnormal compared to those where no troponin was obtained, and even a two-fold increase compared to those in whom troponin was drawn and noted to be normal. Subsequent angiography was five times higher in patients where troponin was drawn and noted to be abnormal - even at very small elevations - and the average

Table 5. 30-day readmission rates for inpatients tested for troponin

\begin{tabular}{|c|c|c|c|c|c|}
\hline & $\begin{array}{l}\text { Troponin } \\
\geq 0.1 \mathrm{ng} / \mathrm{ml}\end{array}$ & $\begin{array}{l}\text { 30-day } \\
\text { Readmission } \\
(\%, \pm 95 \% \mathrm{Cl})\end{array}$ & $\begin{array}{l}\text { Troponin } \\
<0.1 \mathrm{ng} / \mathrm{ml}\end{array}$ & $\begin{array}{l}30 \text { day } \\
\text { Readmission } \\
(\%,+/-95 \% \mathrm{Cl})\end{array}$ & $P$ value \\
\hline All Patients & 3,743 & $562(15.0 \pm 2.8 \%)$ & 10,583 & $1715(16.2 \pm 1.7 \%)$ & 0.09 \\
\hline Type 1 AMI & 1,151 & $124(10.7 \pm 5.2 \%)$ & 36 & 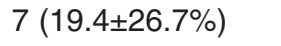 & NS \\
\hline Type 2 AMI & 2,592 & $438(16.9 \pm 3.2 \%)$ & 10,547 & $1708(16.2 \pm 1.6 \%)$ & 0.38 \\
\hline CHF & 476 & $102(21.4 \pm 7.1 \%)$ & 1,362 & 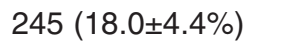 & 0.1 \\
\hline Sepsis & 730 & $93(12.7 \pm 6.3 \%)$ & 1,846 & $262(14.2 \pm 3.9 \%)$ & 0.34 \\
\hline Other & 1,386 & $243(17.5 \pm 4.3 \%)$ & 7,339 & $1201(16.4 \pm 1.9 \%)$ & 0.29 \\
\hline
\end{tabular}

Groups included all patients, those with and without a primary diagnosis type 1 acute myocardial infarction (AMI), and those with primary diagnoses of type 2 AMI including type 2 AMI subsets of CHF, Sepsis and Other (Non-AMI/CHF/Sepsis). (NS = Not statistically significant). 
length of hospital stay was nearly 2 days longer in those with abnormal troponins compared to those without troponin obtained. Moreover, recently published data from our group notes that $\mathrm{TnI}$ testing alone is associated with a $22.6 \%$ increase in hospital charges, and in those without AMI, but values over the 99 th percentile, a $74.2 \%$ increase in charges. ${ }^{3}$ These prior utilization data combined with the current outcomes data reported in this paper highlight the importance of appropriate TnI testing and subsequent clinical decision making based on the results.

As with any large, observational, retrospective work, there are some inherent limitations to the results demonstrated here. Although one of the largest and most recent reports of outcomes in the setting of TnI testing, selection bias with the patient population and inherent testing bias by the physicians limits generalizability to other patient populations. Using ICD codes for categorization of diagnoses in these types of studies is subject to clinician interpretation and familiarity of select codes; however, we found no abnormal diagnostic patterns regarding time or hospital locations. Moreover, there was no propensity toward a specific diagnosis in the "other" category of type 2 AMI patients. Furthermore, the practices and protocols for ordering $\mathrm{TnI}$ results in patients is not standardized across this specific healthcare system, ascertaining sampling biases in these results. Finally, these results are confined to the electronic health system and cannot account for other readmissions or events that might have happened in another hospital system. These data do point to the need for large, prospective studies in which troponin testing is standardized and outcomes vigilantly assessed to develop a predictive model where troponin can be usefully applied toward maximizing patient care.

In conclusion, we demonstrated increased rates of TnI testing among a large cohort of patients admitted across a Texasbased hospital system. Moreover, not only are events such as mortality and readmissions higher among those in which TnI was tested without evidence of type 1 AMI, but increased levels of $\mathrm{TnI}$ above the $99^{\text {th }}$ percentile were also associated with similar increased event rates. These hypothesisgenerating data underscore the need for more prospective work to be done in this field to understand appropriateness of $\mathrm{TnI}$ testing in addition to the prognostic importance of $\mathrm{TnI}$ testing/levels in patients admitted to the hospital with type 2 AMI.

\section{Acknowledgements}

There are no additional contributors, funding sources, or prior presentations for this work. There are no conflicts of interest.

\section{References}

1. Thygesen K, Alpert JS, Jaffe AS, et al. Fourth Universal Definition of Myocardial Infarction (2018). J Am Coll Cardiol. 2018;72(18):2231-2264.
2. Farber A., Suarez K, Slicker K, et al. Frequency of Troponin Testing in Inpatient Versus Outpatient Settings. Am J Cardiol. 2017;119(8):1153-1155.

3. Haneke TW, Widmer RJ, Fry EM, Wilson GM, Michel JB. Hospital Charges Associated with Inpatient Troponin Testing. J Am Coll Cardiol. 2018;72(23 Pt A):2940.

4. Horwich T, Patel J, MacLellan WR, Fonarow GC. Cardiac troponin I is associated with impaired hemodynamics, progressive left ventricular dysfunction, and increased mortality rates in advanced heart failure. Circulation. 2003;108(7):833-838.

5. Jaffe A, Wu AH. Troponin release--reversible or irreversible injury? Should we care? Clin Chem. 2012;58(1):148-150.

6. Thygesen K, Alpert JS, Jaffe AS, et al. Third universal definition of myocardial infarction. J Am Coll Cardiol. 2012;60(16):15811598.

7. Willeit P, Welsh P, Evans JDW, et al. High-Sensitivity Cardiac Troponin Concentration and Risk of First-Ever Cardiovascular Outcomes in 154,052 Participants. J Am Coll Cardiol. 2017;70(5):558-568.

8. Wilson G, Barkley K, Slicker K, Kowal R, Pope B, Michel J. Overuse of Troponin? A Comprehensive Evaluation of Testing in a Large Hospital System. J Hosp Med. 2017;12(5):329-331.

9. Winchester D, Kline K, Estel C, Mahtta D, Taasan S, Peacock FW. Associations between cardiac troponin, mortality and subsequent use of cardiovascular services: differences in sex and ethnicity. Open Heart. 2018;5(1):e000713.

\section{Author Affiliations}

R. Jay Widmer, MD, PhD;* Gibbs Wilson, MD; $;$ Travis Haneke, MD; $;$ Mary Lee, DO;* Jerry Fan, MD $\%$ Arthur Davis, MD $\%$ Ethan Fry, DO; $†$ Kevin Kurian, MD; * Jonathan Urbanczyk, DO;* Jordan Potter, DO; $\uparrow$ Matthew McMahon, MD; $\uparrow$ Bryce Rhodehouse, DO; $\uparrow$ Joon J. Song, PhD; + Jeffrey B. Michel, MD*

*Division of Cardiology, Scott \& White Heart Memorial Hospital, Baylor Scott \& White Health, Temple, Texas, USA

†Division of Internal Medicine, Scott \& White Heart Memorial Hospital, Baylor Scott \& White Health, Temple, Texas. USA tDivision of Biostatistics, Baylor University, Waco, Texas, USA 